\title{
Complexity Reduction of Blind Decoding Schemes Using CRC Splitting
}

\author{
Jonas Eriksson, Reza Moosavi and Erik G. Larsson
}

\section{Linköping University Post Print}

N.B.: When citing this work, cite the original article.

(C)2012 IEEE. Personal use of this material is permitted. However, permission to reprint/republish this material for advertising or promotional purposes or for creating new collective works for resale or redistribution to servers or lists, or to reuse any copyrighted component of this work in other works must be obtained from the IEEE.

Jonas Eriksson, Reza Moosavi and Erik G. Larsson, Complexity Reduction of Blind Decoding Schemes Using CRC Splitting, 2012, 2012 IEEE Global Communications Conference (GLOBECOM), (), , 3731-3736.

http://dx.doi.org/10.1109/GLOCOM.2012.6503697

Postprint available at: Linköping University Electronic Press

http://urn.kb.se/resolve?urn=urn:nbn:se:liu:diva-79226 
Refereed article published in the Proceedings of IEEE GLOBECOM, Dec. 2012. (C) 2012 IEEE. The layout has been revised and minor correction of the affiliations has been done. 


\title{
Complexity Reduction of Blind Decoding Schemes Using CRC Splitting
}

\author{
Jonas Eriksson ${ }^{\ddagger}$, Reza Moosavi ${ }^{\dagger}$ and Erik G. Larsson ${ }^{\dagger}$ \\ $\ddagger$ Ericsson Research Center, Datalinjen 3, SE-581 12, Linköping, Sweden. \\ $\dagger$ Division of Communications Systems, Department of Electrical Engineering, \\ Linköping University, SE-581 83, Linköping, Sweden.
}

\begin{abstract}
Blind decoding, used on control channels of some multi-user wireless access systems, is a technique for achieving adaptive modulation and coding. The idea is to adapt the modulation and coding scheme to the channel quality but instead of signaling the parameters used explicitly, the receiver blindly tries a number of fixed parameter combinations until a successful decoding attempt is detected, with the help of a cyclic redundancy check. In this paper we suggest a new method for reducing the complexity and energy consumption associated with such blind decoding schemes. Our idea is to use a mini-CRC injected early in the data stream to determine if the current decoding attempt is using the correct modulation and coding parameters. We analyze and exemplify the complexity gain of this approach and also investigate the impact of the rearrangement of the CRC scheme in terms of the probability of undetected error. The presented results for the complexity gain are promising and the impact on the error detection capability turns out to be small if any.
\end{abstract}




\section{Introduction}

To achieve higher throughput many wireless multiple access systems deploy adaptive modulation and coding (AMC). The key idea of AMC is to choose the transmission parameters (modulation format, channel code, etc) on the fly based on the instantenauous channel condition [1]. That is, to use higher order AMC parameters whenever the communication link between the transmitter and the receiver is good and vice versa. This adaptive mechanism typically requires a separate signaling of the AMC parameters. Such information is conveyed over dedicated control channels together with other pertinent control information such as scheduling assignments, hybrid automatic repeat request (HARQ) acknowledgments, etc. For these control channels themselves, AMC is also beneficial and indeed used in many solutions. However, instead of signaling the AMC parameters explicitly on yet another control channel, some wireless multiple access systems such as 3GPP Long Term Evolution (LTE) employ so-called blind decoding of their control channels [2].

According to the blind decoding scheme, the transmitter, based on the quality of the communication link, picks one of the possible AMC formats from the AMC pool for the transmission. Prior to the transmission, the transmitter appends a cyclic redundancy check (CRC) to the data which will be used later on by the receiver to retrieve the data. The receiver, upon receiving the data, decodes it using all AMC formats from the pool and evaluates the CRC. If the CRC checks for a decoding attempt, then the receiver assumes that the correct AMC format has been found and that the data is not corrupted.

In LTE, the blind decoding concept also extends to cover the multiple access problem on the control channel, by letting the control data of a specific user have a nonpredetermined position in the control channel data stream, and relying on blind trial and error search by the receiving terminals to solve the multiple access problem [2].

The blind decoding incurs a complexity cost on the receiver side though, since the receiver might need to try many decoding attempts before it finds the correct AMC parameters. Given that the receiver is a mobile terminal with limited battery, any reduction in the decoding complexity incurred by the blind decoding strategy is important.

The methods for reducing the blind decoding complexity can essentially be divided into two separate categories. The first one is search space reduction in which different clever designs are used to reduce the number of blind decoding attempts that a receiver needs to perform in order to retrieve its data. The second one is early decoding termination in which one tries to detect early in the decoding process whether the current blind decoding attempt is performed with the correct set of AMC parameters or not. If not, then the decoding is terminated before completion. The current work belongs to the second category. 


\section{Related Work}

We are only aware of relatively little literature addressing the blind decoding problem. For example, in [3] a method for reducing the search space of the terminals was proposed. The idea is to limit the number of combinations in which the control data blocks may be arranged and located in the time/frequency domain, which in turn results in a search space reduction. In [4], a solution to decrease the number of blind decoding attempts is proposed. The idea is to decode the received data partially according to each combination in the search space and terminate the ones that yield low quality metrics. The quality metric can be cumulative log-likelihood ratios for instance. In [5], a scheme for fast blind identification of channel codes was proposed. The idea is to use the fact that any channel code imposes a certain pattern on the data string, which can be exploited for fast detection of the code.

In [6] a solution to decrease the detection errors of blind decoding in LTE is proposed. The idea therein is to modify the circular buffer used to read out the coded bits, in such a manner that the control information can be decoded unambiguously by the receiving terminals. Manipulation of CRC schemes to enhance detection performance has been considered before in other settings. In [7-9] and further references therein, blind detection of codeword lengths is improved by in some cases reversing the CRC bits or even flipping them according to specific bit patterns.

\section{Contributions}

In this paper we describe and analyze a method for aborting erroneous blind decoding attempts early and hence reduce the complexity and energy consumption of the blind decoding approach. More precisely, we suggest injecting a mini-CRC early in the data stream and use it for early termination of decoding attempts employing the wrong set of coding parameters. The supporting rationale behind this approach is that such decoding attempts will yield errors almost directly in the stream of decoded bits. If the mini-CRC does not check, the current decoding attempt may be aborted, which consequently reduces the complexity and energy consumption of the blind decoding scheme. The detection performance of the scheme is exemplified and its associated complexity reduction is analyzed as well as the modification's impact on the overall error detection capability of the CRC scheme.

The current study limits itself to detection of coding parameters. However, the basic ideas presented herein can most likely be adopted straightforward to address the multiple access problem as well, since an incorrect assumption on data location will produce similar operating conditions for the mini-CRC as an incorrect assumption of AMC parameters. 


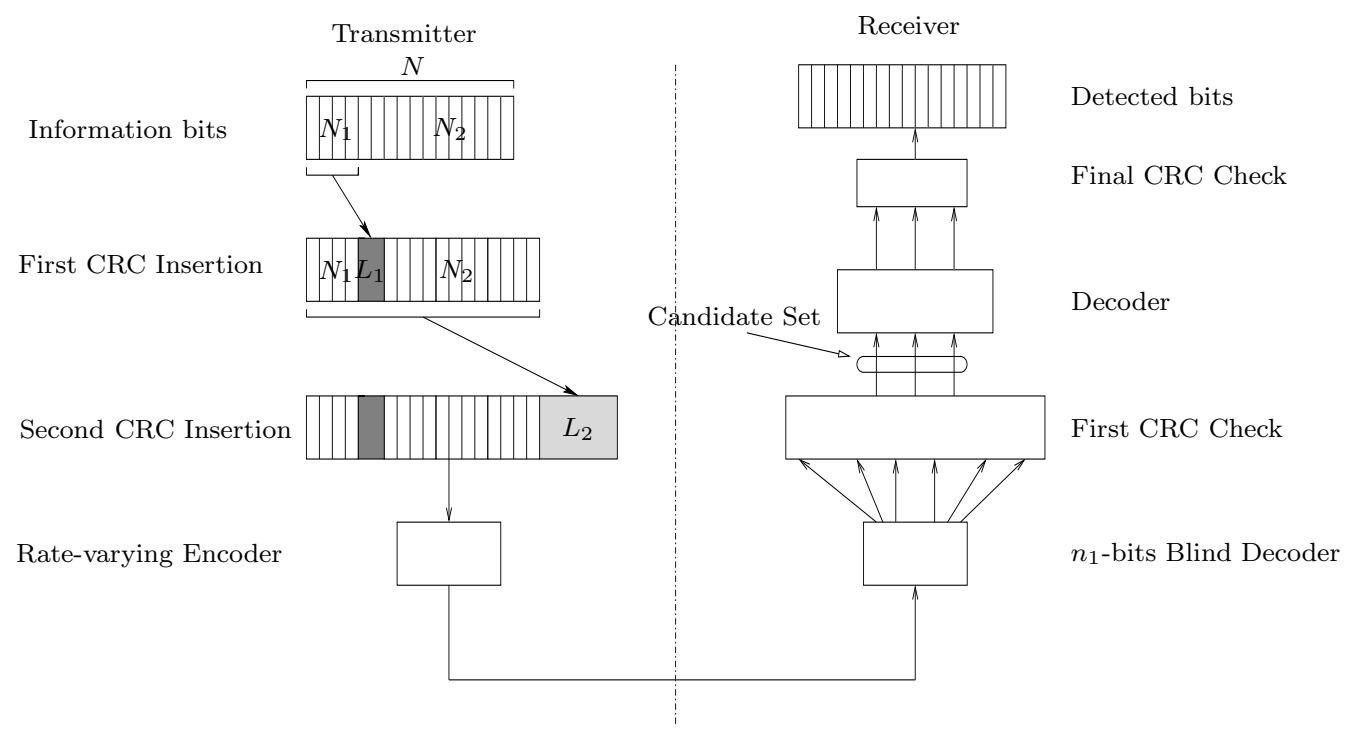

Figure 1: Transmitter and receiver design for the CRC splitting technique. Note that in this figure, it is assumed that the receiver knows the start of the information bits. In cases that the start is unknown, the receiver will consists of a number of such branches in parallel each corresponding to a different starting point.

\section{Proposed Scheme: CRC Splitting}

We proceed by proposing a method for reducing the complexity of the blind decoding process. The basic idea is to use a mechanism such that the terminals do not have to perform a full decoding for each channel code in the AMC pool. That is, after blindly decoding a small subset of incoming bits, the terminal will decide on candidate codes and continue the process of decoding using this candidate set. This will in turn decrease the overall computational complexity.

Figure 1 illustrates the procedure for the proposed scheme. Instead of attaching the $L$ bit CRC at the end of the information bits (as in the conventional approach), the $N$-bit message is partitioned into two parts, of lengths $N_{1}$ and $N_{2}$, say. A shorter $L_{1}$-bit mini-CRC is attached after the first part and a second, longer $L_{2}$-bit CRC is inserted after the second part. To preserve the code rate of the original system, we choose $L=L_{1}+L_{2} \cdot{ }^{1}$ The second CRC comprises parity bits obtained from both parts of the message with parity on parity.

After blindly decoding the first $n_{1}=N_{1}+L_{1}$ information bits for all possible codes, the terminal checks whether the mini-CRC is satisfied. If yes, the terminals

\footnotetext{
${ }^{1}$ Alternatively, the mini-CRC can be added to the data block, allowing for preservation of the error detection property, but this increases the length of the block and reduces the rate of the transmission.
} 
considers the corresponding code to be a "candidate" for further consideration. If the CRC does not pass for a certain code, then the terminal will not add it to the candidate set, and not further consider this code. Note that for different codes, $n_{1}$ information bits correspond to different number of channel symbols (since the rates of the codes are different). Once the terminal has tried to decode the mini-CRC for all codes, it proceeds to performing a full decoding attempt only for the codes in the candidate set. Preferably, the CRC checks should be chosen so that the number of codes in the candidate set is small (or equal to one, most of the time). The order in which to try the candidates in the candidate set is of course important since we preferably want to find the correct candidate directly. Although one can envision interesting strategies based on, for instance, a comparison of the absolute values of the decoding metrics after the decoding of the first $n_{1}$ bits, we defer from venturing further into that field of study in this work.

An underlying assumption of the proposed scheme is a decoding process which can pause its operation and produce a partial decoding result. Specifically, we want it to produce a reliable decoding of only the first $n_{1}$ information bits of the codeword. This requires the use of a decoding algorithm which works linearly along the codeword, such as the Viterbi algorithm. Thus in the numerical illustrations presented in Section 6, we suggest using the Viterbi algorithm and halting its operation after traversing $n_{1}+\Delta$ sections of the associated trellis, where $\Delta$ is a small number on the order of the constraint length of the convolutional code in question. At that position the state displaying the best metric is used as a starting point for backtracking through the trellis to produce a reliable partial decoding result for the first $n_{1}$ bits. Given that the adaptive modulation and coding scheme of the overall system is working appropriately, it should be possible to use a common $\Delta$ for all considered coding schemes and signal-to-noise ratios (SNRs).

\section{Split-CRC Error Detection Performance}

Reducing the original CRC by a number of bits and then inserting them earlier into the data stream in the form of an additional mini-CRC, of course changes the overall error detection performance of the CRC scheme. In order to grasp the impact of CRC-splitting on error detection performance one can study the performance over a binary symmetric channel (BSC). Under these assumptions the probability of undetected error can be expressed as

$$
P_{u e}=\sum_{i=1}^{n} A_{i} p^{i}(1-p)^{n-i}
$$

where $n$ is the length of the codeword (data packet including CRC), $p$ is the crossover probability of the BSC and $\left\{A_{i}\right\}_{i=1}^{n}$ it the weight distribution of the codewords formed by the CRC calculation (the CRC-code) [10]. If we determine the generator 


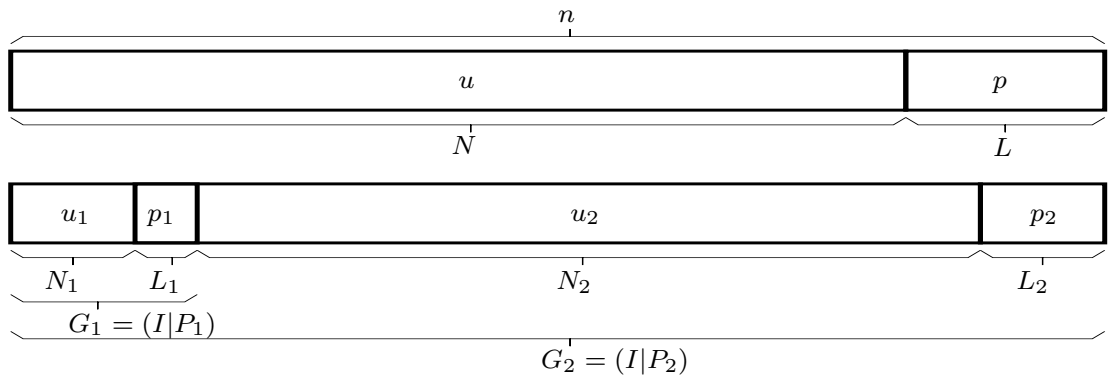

Figure 2: Conventional CRC scheme (top) and the suggested CRC-splitting scheme (bottom) with associated generator matrices.

matrix of our split-CRC scheme viewed as a binary linear error detecting code, we can obtain its weight distribution and use (1) to calculate the probability of undetected error.

Let us assume that the mini-CRC is $L_{1}$ bits long and calculated using the first $N_{1}$ bits of the data stream. The data bits together with the parity bits constitutes a codeword in the binary linear code with $N_{1} \times\left(N_{1}+L_{1}\right)$ generator matrix $G_{1}=$ $\left(I \mid P_{1}\right)$. Similarly $G_{2}=\left(I \mid P_{2}\right)$ is the $\left(N_{1}+L_{1}+N_{2}\right) \times\left(N_{1}+L_{1}+N_{2}+L_{2}\right)$ generator matrix of the code corresponding to taking the $L_{2}$ bit CRC of the $N_{1}+L_{1}+N_{2}$ first bits of the data stream (parity on parity). Let $u_{1}$ and $u_{2}$ be the $N_{1}$ bit and $N_{2}$ bit row vectors of information bits. Figure 2 gives a graphical illustration of the situation. The codeword created in the split CRC scheme can be written as

$$
c=\left[u_{1}, u_{1} P_{1}, u_{2},\left[u_{1}, u_{1} P_{1}, u_{2}\right] P_{2}\right]=[u 1, u 2] \tilde{G}
$$

where $\tilde{G}$ is the overall generator matrix. It can be shown that

$$
\tilde{G}=\left[\begin{array}{cccc}
I_{N_{1}} & P_{1} & 0 & P_{2}^{(1)}+P_{1} P_{2}^{(2)} \\
0 & 0 & I_{N_{2}} & P_{2}^{(3)}
\end{array}\right]
$$

where we have used the following partitioning of $P_{2}$ :

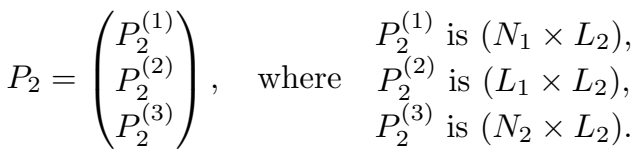

Via simple column reordering, we can get an equivalent generator matrix on systematic form as

$$
G=\left[\begin{array}{cccc}
I_{N_{1}} & 0 & P_{1} & P_{2}^{(1)}+P_{1} P_{2}^{(2)} \\
0 & I_{N_{2}} & 0 & P_{2}^{(3)}
\end{array}\right] .
$$




\subsection{Numerical Examples — Error Detection}

Using expressions (2) and (3) as input, we have done brute force calculations of the weight distributions of some example versions of the CRC-code formed by the split-CRC scheme. More precisely, we have derived the generator matrices as above and plugged them into the MAGMA computational algebra system [11], which has efficient standard routines for calculation of the weight distribution of a linear code, given the generator matrix. We study the cases when $n=66,116$ and 216 for $L_{1}=4$ and $L_{2}=12$. The comparison is made with a conventional $L=16$ bit CRC scheme. The generator polynomials used for the CRCs are the standard polynomials CRC-4-ITU, CRC-12 and CRC-16-CCITT listed below.

$$
\begin{aligned}
g_{4}(X) & =X^{4}+X+1 \\
g_{12}(X) & =X^{12}+X^{11}+X^{3}+X^{2}+X+1 \\
g_{16}(X) & =X^{16}+X^{12}+X^{5}+1 .
\end{aligned}
$$

Assuming that the BSC stems from a BPSK AWGN channel, the numerical evaluation of (1) is presented in Figures 3, 4 and 5. From the curves it is apparent that in the moderate to high SNR regime the impact on the overall error detection performance when splitting the CRC into two parts seem to be negligible. For the low SNR regime there is a varying impact on performance. A properly chosen insertion point can even give a better overall error detection performance than the original 16 bit scheme for short block lengths. It is however apparent that choosing the insertion point later into the data stream is beneficial for the error detection performance. This fact strives in the opposite direction of our wish to terminate decoding as soon as possible and thereby have an early insertion point. A trade off is needed, but for the studied cases there seem to be plenty of room for doing this trade off without compromising neither of the two aspects to much, especially for reasonably high SNRs.

It is perhaps worth noting that the peak in the probability of undetected error displayed in the curves, stems from the fact that the typical error pattern weight at those SNRs matches the minimum distance of the CRC-code in question. Error detecting codes not displaying this property are known as proper codes and are typically not formed by standard CRC checks but constitute a complete research field of their own.

\section{Split-CRC Code Detection Performance}

The capability of the proposed scheme in terms of discriminating between different coding schemes, is what governs its feasibility and the associated complexity 


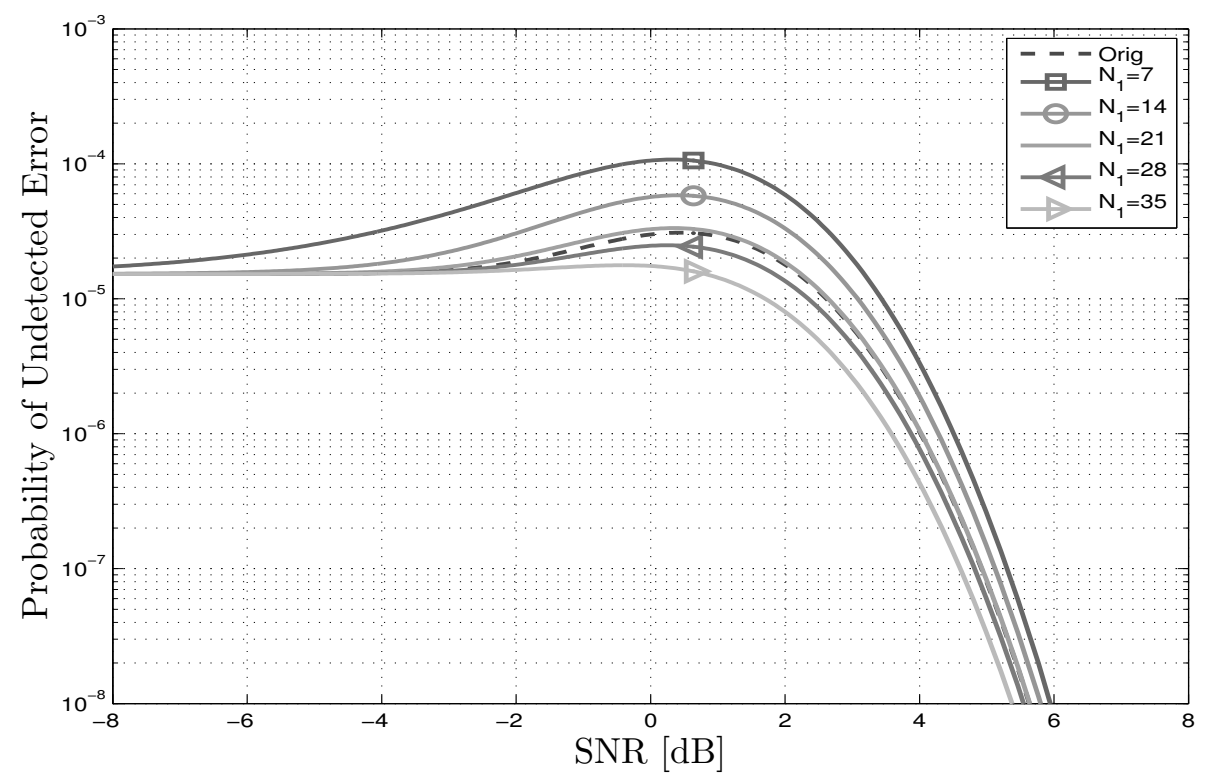

Figure 3: Comparison of overall error detection performance for different mini-CRC insertion points for $N+L=66$.

reduction. In order to grasp this capability, we define three events: (i) detection, (ii) missed detection and (iii) false alarm. By detection we mean that the checking of the mini-CRC turns out positive for the code that is actually used for the encoding (true code), regardless whether it turns out positive for any other code or not. This means that we find a positive outcome of our proposed scheme if the true code is in the candidate set for further processing. As a natural consequence we also keep track of the size of this set, since it has impact on the overall complexity. Missed detection is simply the complementary event to detection. A false alarm is declared if the checking of the mini-CRC produces a non-empty candidate set in which the true code is not included. Also in this case we keep track of the size of the candidate set.

It is worth noting that the usage of a CRC for error detection performance is typically in a situation where one can expect the number of errors to be relatively small. As the number of errors increase, there is an increase (at least initially) in the probability of undetected error. In the blind decoding scenario, we know that we will produce a high number of errors when we decode using the wrong assumption on the code parameters. There is then in principle the possibility that this will effect the probability of undetected error so much that it affects the overall performance of the proposed scheme. 


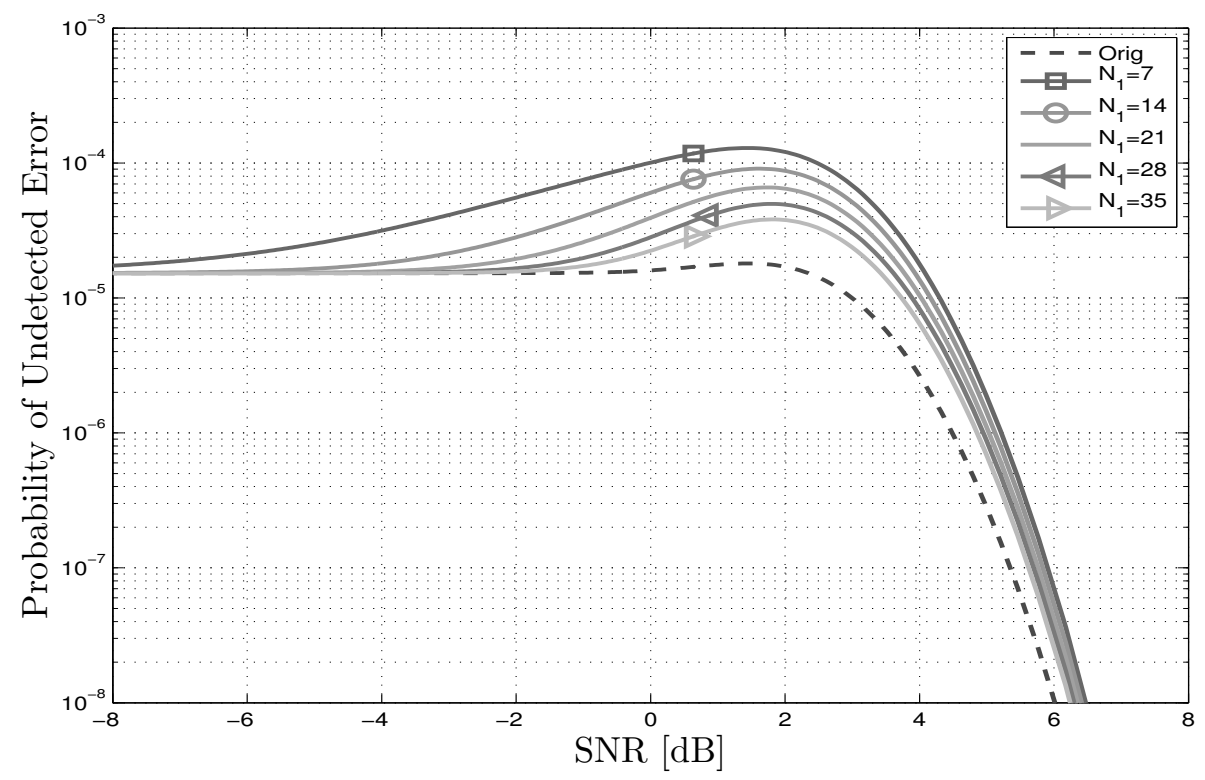

Figure 4: Comparison of overall error detection performance for different mini-CRC insertion points for $N+L=116$.

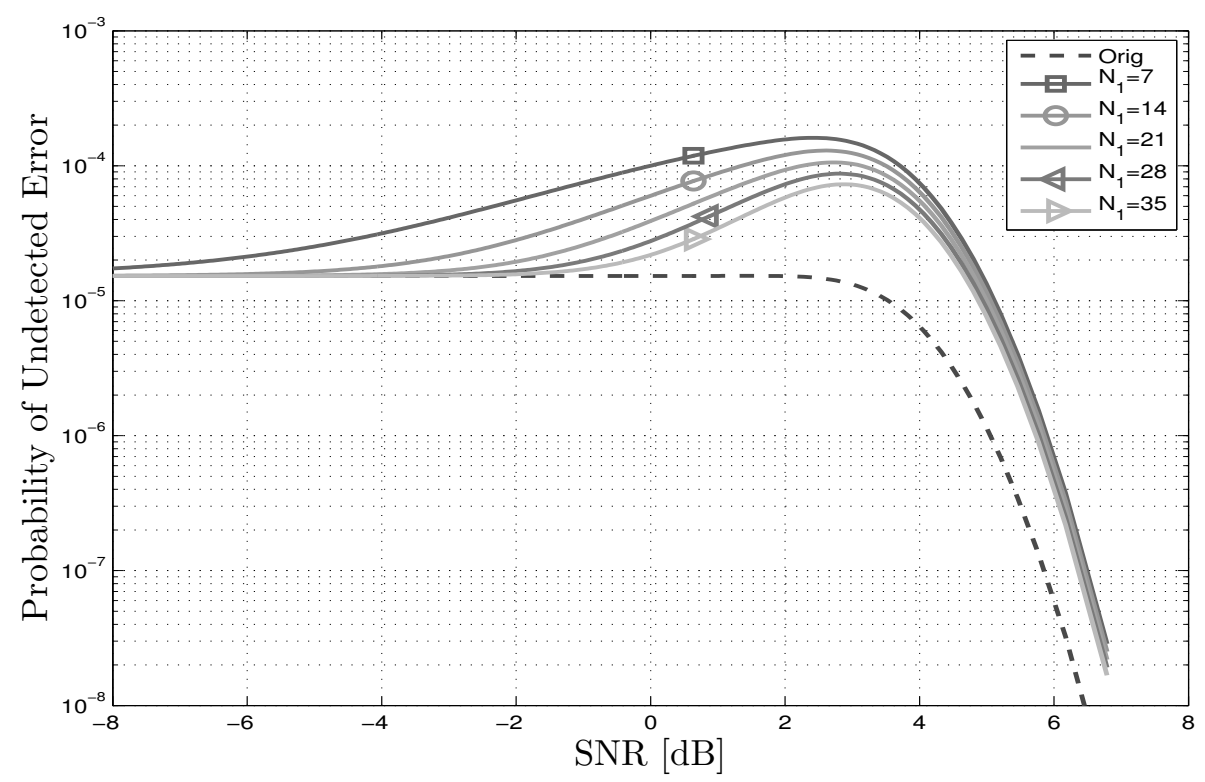

Figure 5: Comparison of overall error detection performance for different mini-CRC insertion points for $N+L=216$. 


\subsection{Numerical Examples — Code Detection}

In order to convey an insight into the properties of our proposed scheme, we have produced curves for the probability of missed detection $P_{\mathrm{MD}}$ and probability of false alarm $P_{\mathrm{FA}}$ respectively, for an example scenario using Monte Carlo simulations. We have also obtained curves for the average size of the candidate set for both the detection event $\left(N_{\mathrm{D}}\right)$ as well as for the false alarm event $\left(N_{\mathrm{FA}}\right)$.

The choice of channel model and channel codes in the AMC pool does not change the principles of our scheme. Therefore, in our example setup, we use somehow arbitrarily a rate $1 / 2$ (with generators " 5375 ") constraint length $K=6$ terminated convolutional code combined with $N_{\mathrm{C}}=6$ different puncturing matrices as our AMC pool, and we use a BPSK AWGN channel model. The resulting codes have non-terminated rates of $2 / 3,3 / 4,4 / 5,5 / 6,6 / 7$ and $7 / 8$ respectively. We assume that the codes all have equal probability of being used for the encoding. This is of course an oversimplification for a system employing AMC, which would make the choice highly SNR dependent. However, we defer from deriving a more complex and involved model including the AMC and instead settle for the fact that our assumption on equally probable coding schemes will yield slightly pessimistic (i.e. higher) values for $P_{\mathrm{MD}}$ and $P_{\mathrm{FA}}$. Further we use $N=50, N_{1}=15, L_{1}=4$ and 8 , $L_{2}=12$ and 8 and $\Delta=5$.

The simulation setup follows the basic principles described in Section 4. For each channel code from the AMC pool and for each SNR point that we wish to evaluate, we make a Monte Carlo simulation. For each Monte Carlo run, we create a random bit string and split it into two parts according to the parametric settings to be evaluated. Then CRC parity bits are calculated and added to the first and second parts according to the proposed scheme in Section 4. The channel code is then used to encode the CRC-embedded information bits which subsequently are modulated and transmitted over a simulated communication channel. After demodulation into soft bits the blind decoding commences. For each code in the AMC pool, the Viterbi algorithm is run on the received soft bits and halted to produce a partial decoding result according to the method described in Section 4. Code detection is then done by checking the $L_{1}$-bit CRC. The detection result for each code in the AMC pool is noted and used to produce one Monte Carlo sample for each of the events defined in the beginning of Section 6. After completing Monte Carlo simulations for all codes in the AMC pool - for a given SNR - we average over the number of runs and over the codes to obtain values for $P_{\mathrm{MD}}, P_{\mathrm{FA}}, N_{\mathrm{D}}$ and $N_{\mathrm{FA}}$ respectively.

The simulation results are shown in Figures 6 and 7, where Figure 6 illustrates good detection performance for the proposed scheme as both $P_{\mathrm{MD}}$ and $P_{\mathrm{FA}}$ falls rapidly with SNR. Using the longer mini-CRC mainly affects $P_{\mathrm{FA}}$ which is reduced. This is due to the fact that the two combined error generating processes - decoding with incorrect parameters and the AWGN - has less probability of hitting a codeword at random in the CRC code (and thus causing a false alarm), when it uses more parity 


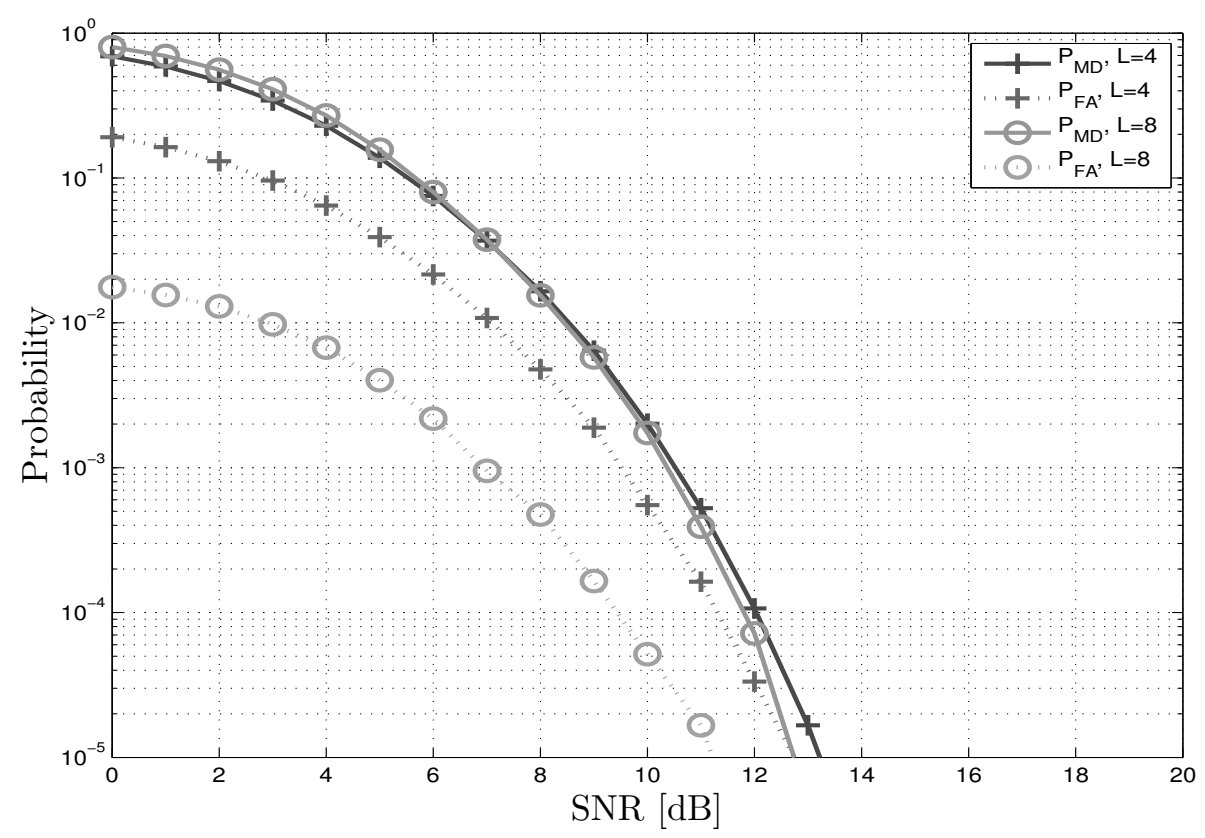

Figure 6: Empirical probability of Missed Detection $P_{\mathrm{MD}}$ and False Alarm $P_{\mathrm{FA}}$ as a function of signal-to-noise ratio for $L_{1}=4$ and 8 .

bits. On the other hand, the missed detection event depends on the error correction capability of the actual channel code that was used to encode the data, and that only differs slightly when the codeword length is changed with 4 bits to accommodate $L_{1}=4$ or $L_{1}=8$. From Figure 7 , it is apparent that the discrimination is very good, since we have $N_{\mathrm{D}}$ and $N_{\mathrm{FA}}$ very close to unity, with slightly better performance for the stronger $L_{1}=8$ code. We have also included the upper 99 th percentile for the empirical distribution of both $N_{\mathrm{D}}$ and $N_{\mathrm{FA}}$ as a complement to their respective averages. In $99 \%$ of the cases, $N_{\mathrm{D}}$ and $N_{\mathrm{FA}}$ are below these curves. It is apparent that the distribution for $N_{\mathrm{D}}$ is more narrow than that of $N_{\mathrm{FA}}$ and that in a majority of cases the decoding process will continue with only one or possibly two coding schemes in the candidate set.

\section{Complexity reduction}

The complexity reduction offered by the proposed scheme is a linear reduction of each blind decoding attempt. The reduction stems from the fact that we do not need to proceed with a decoding attempt after the pause - where the mini CRC is evaluated - if this evaluation turns out to be negative. In order to make a rough 

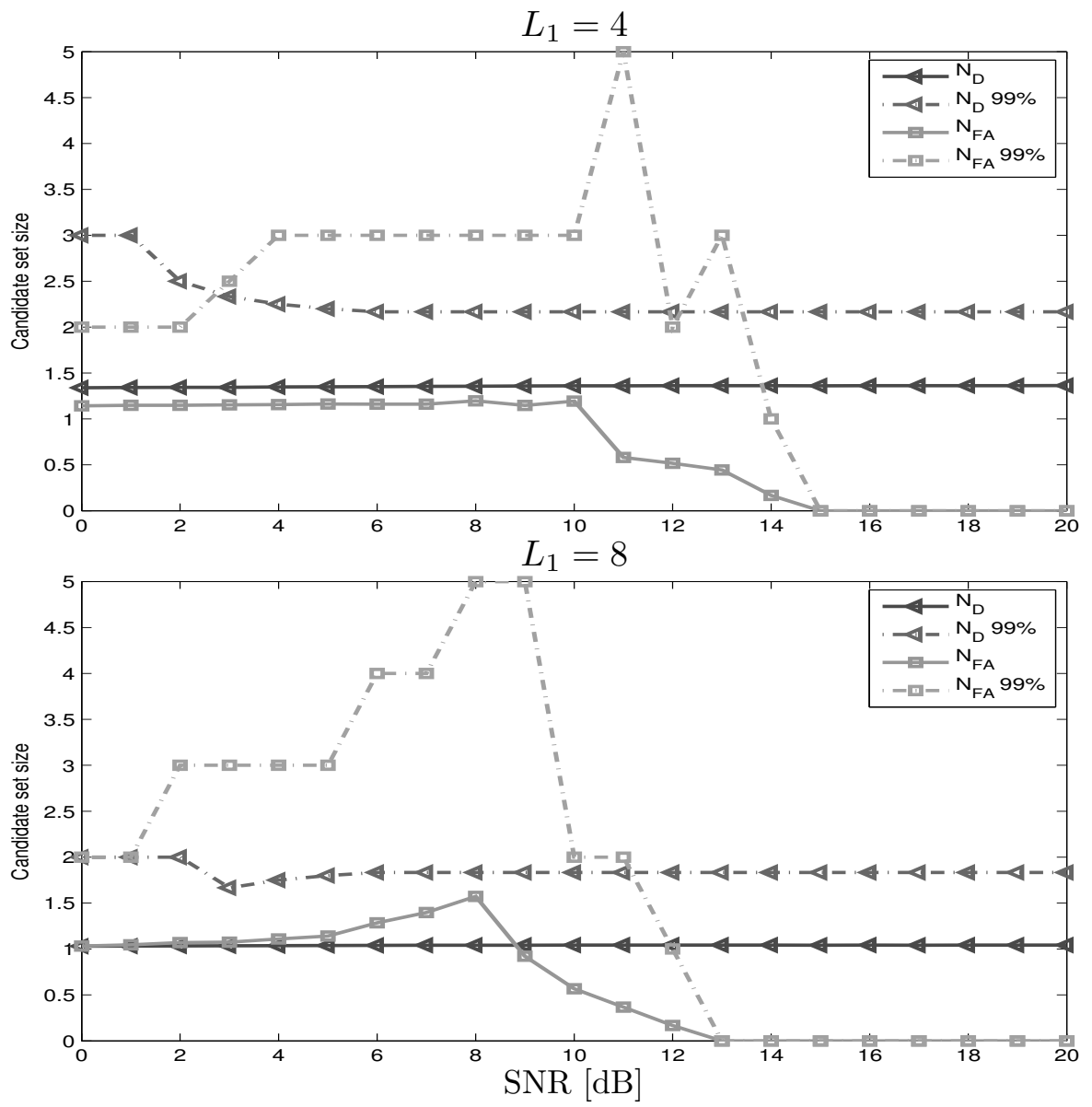

Figure 7: Empirical values for candidate set sizes in case of detection $\left(N_{\mathrm{D}}\right)$ and false alarm $\left(N_{\mathrm{FA}}\right)$. Respective $99 \%$ upper percentiles are also plotted. 
study of the complexity gain of our proposed scheme we make some assumptions. Consider the illustrative example above with Viterbi decoding. Let us stipulate that the complexity of CRC evaluations of both the original and the CRC splitting scheme are on equal level and small compared to the Viterbi decoding. We then proceed by noting that the original blind decoding scheme would have a running complexity that requires $\mathcal{O}\left(2^{K-1} n N_{\mathrm{C}}\right)$ operations, stemming from the full Viterbi decoding of $N_{\mathrm{C}}$ length $n$ terminated convolutional codes with constraint length $K$. For our proposed scheme, we say that the missed detection event triggers a full run of the original blind decoding scheme. The false alarm event, which is a sub class of the missed detection event, is assumed to not entail any extra complexity to be handled compared to a missed detection. The underlying rationale for this assumption is that we assume that the false alarm is revealed after the full decoding of the false candidates using the overall $\mathrm{CRC}$, and that this triggers full decoding of the remaining codes. Given the above assumptions, we can say that the running complexity of our proposed scheme is $\mathcal{O}\left(2^{K-1} n_{1} N_{\mathrm{C}}+P_{\mathrm{MD}} 2^{K-1}\left(n-n_{1}\right) N_{\mathrm{C}}+(1-\right.$ $\left.\left.P_{\mathrm{MD}}\right) 2^{K-1}\left(n-n_{1}\right) N_{\mathrm{D}}\right)$. The three terms within the parentheses come from (i) the decoding of all $N_{\mathrm{C}}$ codes up till the length $n_{1}$, (ii) the full decoding of all codes in the event of a missed detection, and (iii) the continued decoding of on average $N_{\mathrm{D}}$ codes in the event of successful detection.

By expressing $n_{1}$ as a fraction of $n$ by $n_{1}=\alpha n$ and similarly using $N_{\mathrm{D}}=\beta N_{\mathrm{C}}$, we can write this as $\mathcal{O}\left(2^{K-1} n N_{\mathrm{C}}\left(P_{\mathrm{MD}}(1-\alpha)(1-\beta)+\alpha+\beta-\alpha \beta\right)\right)$. We can note that the complexity scaling factor introduced by CRC splitting essentially follows $P_{\mathrm{MD}}$ as a function of SNR since both $\alpha$ and $\beta$ (roughly) are constants in that respect. This also means that for the high SNR case $\left(P_{\mathrm{MD}} \longrightarrow 0\right)$ we have a running complexity of $\mathcal{O}\left(2^{K-1} n N_{\mathrm{C}}(\alpha+\beta-\alpha \beta)\right)$, pointing to a substantial complexity reduction for small values of $\alpha$ and $\beta$. For our studied example above with $L_{1}=4$, we have $\alpha \approx 0.288$ and $\beta \approx 0.167$, yielding a complexity scaling factor of about 0.407 . Also for the studied scenario with $L_{1}=8$, we have $\alpha \approx 0.348$ and $\beta \approx 0.217$, resulting in a complexity scaling factor of about 0.490 .

\section{Conclusions}

We have proposed a method that reduces the complexity of the blind decoding processes in systems such as LTE. The scheme entails manipulation of the CRC error detection scheme inherent in those types of processes. Specifically, it involves splitting off a small part of the original CRC and placing it early in the data block. The mini-CRC part is used for early termination of erroneous blind decoding attempts. The presented results for the detection performance of this CRC splitting approach and its associated complexity gain point to the possibility of substantial improvements. Our analysis also showed that the new type of CRC arrangement offers an overall error detection performance which differs only slightly from that offered by the conventional approach at reasonable high SNRs. 


\section{References}

[1] A. J. Goldsmith and S. G. Chua, "Adaptive coded modulation for fading channels", IEEE Trans. Commun., vol. 46, no. 5, pp. 595-602, May 1998.

[2] E. Dahlman, S. Parkvall and J. Sköld, 4G LTE/LTE-Advanced for Mobile Broadband, 1st edition Academic Press, 2011.

[3] D. P. Malladi, J. Montojo and S. Sarkar, Methods and systems for PDCCH blind decoding in mobile communications, United States Patent Application Publication 2009/0168922, Jul. 2, 2009.

[4] A. Reial, L. Andersson, HS-PDSCH blind decoding, United States Patent Application Publication 2009/003301, Jan. 1, 2009.

[5] R. Moosavi and E. G. Larsson, "A fast scheme for blind identification of channel codes," in Proc. of IEEE Global Telecommunications Conference (GLOBECOM), Dec. 2011.

[6] J. F. Cheng, System and method for removing PDCCH detection errors in a telecommunications network, United States Patent Application Publication 2010/0050059, Feb. 25, 2010.

[7] S. Shien, P.-N. Chen and Y. S. Han, Flip CRC Modification for Message Length Detection, IEEE Trans. Comm., vol. 55, no. 9, pp. 1747-1756, Sep. 2007.

[8] S. Shien, S.-T. Kuo, P.-N. Chen and Y. S. Han, Strategies for Blind Transport Format Detection using Cyclic Redundancy Check in UMTS WCDMA, ICWMCNC2005, vol. 2, pp. 44-50, Aug. 2005.

[9] S. Shien, P.-N. Chen and Y. S. Han, A Novel Modification of Cyclic Redundancy Check for Message Length Detection, ISITA2004, pp. 178-183, Oct. 2004.

[10] S. Lin and D. J. Costello, Jr., Error Control Coding Fundamentals and Applications, 2nd edition Pearson Education International 2004.

[11] http://magma.maths.usyd.edu.au 